\title{
Incidencia y letalidad por infarto agudo del miocardio en Chile: 2001-2007
}

\author{
CAROLINA NAZZAL ${ }^{\text {a }}$ FAUSTINO TOMÁS ALONSO
}

\section{Incidence and case fatality due to acute myocardial infarction in Chile in the period 2001-2007}

Background: Acute myocardial infarction (AMI) causes $73.6 \%$ of coronary heart disease (CHD) deaths in Chile. Aim: To estimate the incidence and case fatality of AMI and analyze their trends between 2001-2007. Material and Methods: A timeseries study analyzing all cases of AMI (according to the International Classification of Diseases (ICD)-10, I21 code), registered in the National Hospitalizations and Death databases. Annual incidence rates and case fatality by sex and age groups were calculated. The direct method was used to standardize rates by age, using the World Health Organization 2000 Population. Prais-Winsten regression models were used to evaluate trends, expressed as relative change. Results: Between 2001 and 2007, we estimated that 83,754 cases of AMI occurred. Standardized annual incidence rate was 74.4 per 100,000 inhabitants (98.0 in men and 51.0 in women). Incidence rates increased by $34 \%$ in individuals $<45$ years of age and $9.2 \%$ in the group 55-64 years ( $p<0.001$, both). Total case fatality was $49.5 \%$ (45.4\% in men and $57.2 \%$ in women; $p<0.001)$, and its trend analysis showed a significant annual reduction of $1.2 \%$ in men and $0.81 \%$ in women. In-hospital case fatality was $14.2 \%(11.3$ and $20.4 \%$ in men and women, respectively; $p<0.001)$. There was a significant annual reduction of mortality (0.57 and $1.01 \%$ in men and women, respectively $(p<0.05)$. Conclusions: The incidence of AMI was stable in the whole population, but increased in younger age groups. Total and in-hospital case-fatality decreased. Despite the greater reduction of case fatality in women, they still have a higher risk of dying while in hospital.

(Rev Med Chile 2011; 139: 1253-1260).

Key words: Cause of death; Chile; Incidence; Mortality; Myocardial infarction.

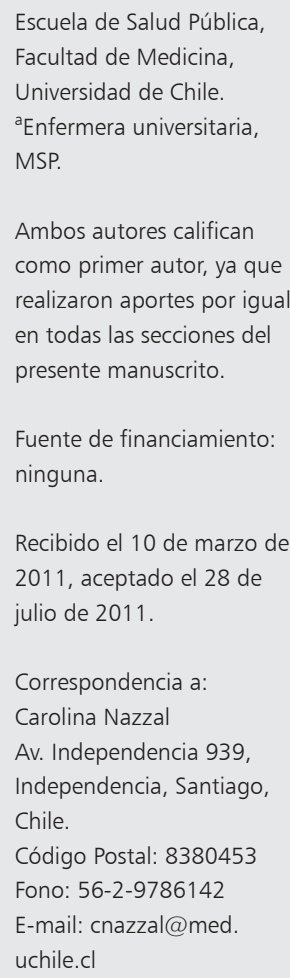

L a enfermedad coronaria (EC), responsable de $9,5 \%$ del total de defunciones en Chile, es la primera causa de muerte en hombres y la segunda en mujeres, después del accidente cerebrovascular ${ }^{1}$. Setenta y tres coma seis porciento de las muertes por EC son debidas a infarto agudo del miocardio (IAM) ${ }^{2}$. En Chile, así como en países de Europa Central y en Estados Unidos de Norteamérica, la mortalidad por cardiopatía coronaria ha disminuido en las últimas décadas ${ }^{3-6}$. Sin embargo, a pesar de esta reducción, se proyecta que seguirá siendo un problema prioritario de salud pública debido a su gran magnitud'. Según la evaluación realizada por el Ministerio de Salud chileno el año 2010, la reducción en la mortalidad por EC entre 1998 y 2007 fue de $24 \%$, siendo la tasa ajustada por edad 48,2 x 100.000 habitantes al final del período 6 . La reducción observada en las tasas de mortalidad por EC puede explicarse por una disminución en la incidencia y/o por un aumento en la sobrevida de los pacientes ${ }^{8}$. Los cambios en la incidencia podrían responder a mejor control de los factores de riesgo cardiovascular en la población, producto de estrategias de prevención primaria y secundaria. Por otro lado, la optimización del tratamiento, así como las estrategias de prevención secundaria, 
aumentan la sobrevida de pacientes coronarios, contribuyendo de este modo a la disminución de la mortalidad ${ }^{4,9,10}$.

Para el estudio de la incidencia y la letalidad de IAM se requiere información sobre el número de casos no fatales y fatales que ocurren anualmente en el país. En Chile, el Departamento de Estadísticas e Información en Salud del Ministerio de Salud (DEIS) proporciona la información acerca de las muertes según causa específica, pudiendo determinar de esta forma los casos fatales de IAM. Sin embargo, no se dispone de un registro de incidencia que proporcione la información de los casos no fatales. El objetivo de este trabajo es estimar la incidencia y letalidad de IAM en Chile y analizar su tendencia entre los años 2001 y 2007. Esta información puede ser de utilidad en la planificación de las políticas públicas del país.

\section{Materiales y Métodos}

Se realizó un estudio de series de tiempo que incluyó todos los casos de IAM ocurridos entre enero de 2001 y diciembre de 2007, identificados de las bases de datos de mortalidad y egresos hospitalarios del DEIS para los años mencionados. Fueron seleccionados los casos de IAM fatales y no fatales según el código I21 de la décima revisión de la Clasificación Internacional de Enfermedades $(\mathrm{CIE} 10)^{11}$. Se definió como caso un primer episodio de IAM o un episodio recurrente de IAM. Para determinar el cambio de incidencia o de letalidad en grupos específicos, se estratificó la población según sexo y tramos de edad en: menores de 45 años, 45 a 54 años, 55 a 64 años, 65 a 74 años y 75 o más años.

\section{Análisis estadístico}

Se realizó el análisis descriptivo evaluando la distribución de las variables. La edad fue descrita en forma continua con la mediana y los percentiles 25 y 75 y luego categorizada en los grupos mencionados anteriormente. Las variables categóricas fueron descritas con frecuencias absolutas y relativas. Las comparaciones se realizaron mediante test de Mann-Whitney para variables continuas y $\chi^{2}$ para variables categóricas.

Se calcularon los siguientes parámetros para cada año del período estudiado:

I) Tasa de incidencia: número de casos de IAM (primeros o recurrentes) por 100.000 habitantes.
El numerador de la tasa correspondió a la suma de los siguientes eventos:

a) hospitalizaciones por IAM en pacientes que egresaron vivos (se considera hospitalización a aquel caso que, al menos, permaneció una noche en el hospital); $b$ )muertes por IAM en pacientes hospitalizados; $c$ )muertes por IAM ocurridas en servicios de urgencia adosados a hospitales, pero que no fueron hospitalizados; $d$ )muertes por IAM extrahospitalarias, es decir, las ocurridas en el domicilio u otro lugar (que incluye servicios de atención primaria de urgencia, postas rurales y consultorios, de acuerdo a la definición utilizada en el certificado de defunción).

El cálculo de tasas crudas de incidencia utilizó como denominador la población total estimada al 30 de junio de cada año ${ }^{12}$. Con el fin de evaluar la tendencia, las tasas crudas fueron estandarizadas por edad mediante método directo ${ }^{13}$, utilizando como población estándar la población mundial de la OMS del año $2000^{14}$.

II) Letalidad: se estimó la letalidad total e intrahospitalaria. La primera considera la totalidad de los casos de IAM registrados y corresponde a la proporción de estos que fallecieron tanto dentro como fuera del hospital. La letalidad intrahospitalaria corresponde a la proporción de casos fallecidos del total de casos de IAM que alcanzan a ser hospitalizados.

Los indicadores anteriores se presentan según sexo y edad.

La tendencia de las tasas de incidencia y letalidad fue evaluada mediante un modelo de regresión de Prais-Winsten $(\mathrm{PW})^{15}$, comprobando sus supuestos. Este método, similar a una regresión lineal, se utiliza cuando las variables son dependientes del tiempo y los errores están correlacionados. Su resultado se expresa en un coeficiente que expresa en este caso el cambio absoluto en las tasas de incidencia o letalidad por cada año. Además, se utilizaron los valores predichos mediante el modelo para estimar el número de casos incidentes hasta el año 2015.

El nivel de significación considerado fue 5\% y los análisis estadísticos fueron realizados con el software STATA $11.2^{16}$.

\section{Resultados}

Entre el año 2001 y 2007 se estimó que ocurrieron 83.754 casos de IAM con un promedio 


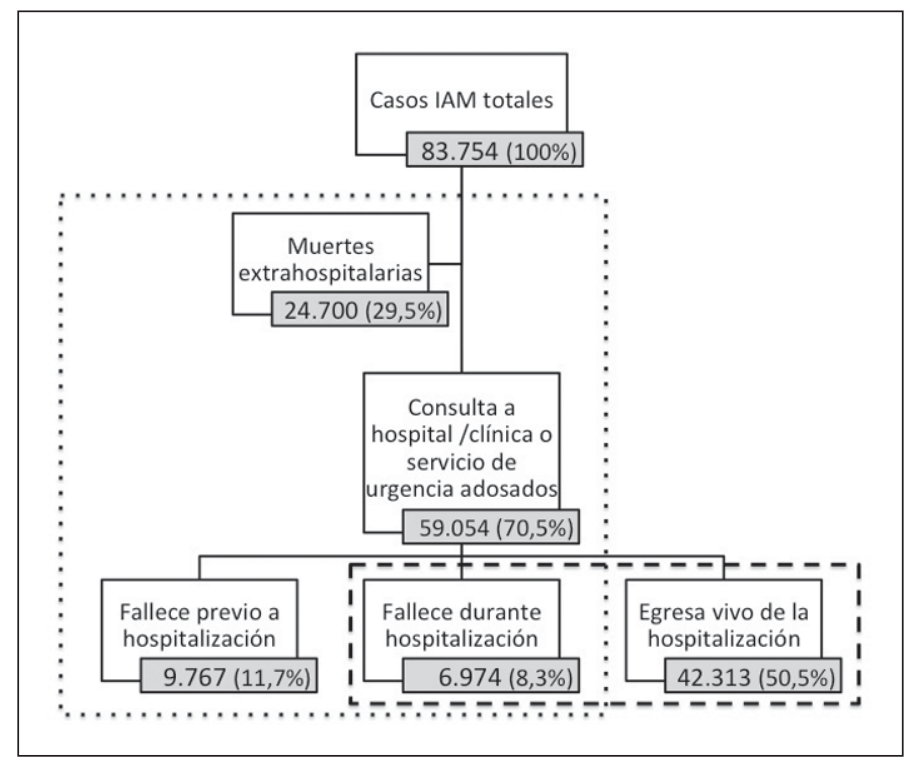

Figura 1. Composición de la población en estudio de casos de infarto agudo del miocardio en Chile, 2001-2007. La línea punteada delimita los casos de IAM obtenidos a partir de las bases de datos de defunciones. La línea segmentada delimita a los casos de IAM obtenidos de las bases de datos de egresos hospitalarios. anual de 11.964 casos. Los hombres representaron 65,3\%.

En la Figura 1 se muestra la composición de la población del estudio de acuerdo a las bases de datos utilizadas. Del total de episodios de IAM, 70,5\% (59.054) consultó en un hospital/clínica o servicio de urgencia adosado, de estos últimos, 83,5\% (49.287) estuvo, al menos, una noche en el hospital; esto equivale a decir que 58,8\% del total de episodios de IAM fueron hospitalizados. Al evaluar según sexo, se encontraron diferencias significativas en el porcentaje de pacientes que consultaron en un hospital o clínica $(73 \%$ en hombres vs 68,5\% en mujeres; $\mathrm{p}<0,001$ ); así como en el porcentaje de pacientes que consultaron y fueron hospitalizados $(84,8 \%$ de los hombres vs $80,6 \%$ de las mujeres $\mathrm{p}<0,001)$.

Respecto a la edad, la mediana en los hombres fue 65 años (p25-p75: 55-75), mientras que en las mujeres, ésta fue 74 años (64-83); p < 0,0001. Al comparar los pacientes que no fallecieron vs los fallecidos, también se observó una diferencia en la edad; en los primeros fue 62 años (53-72) mientras que en los segundos, 74 años (64-82); p $<0,0001$.

La tasa cruda promedio de incidencia fue 74,4 casos por cada 100.000 habitantes $(98,0 \mathrm{x}$ 100.000 en hombres y 51,0 x 100.000 en mujeres). La evolución temporal de las tasas de incidencia estandarizadas por edad para hombres y mujeres se muestra en la Figura 2. En ella se observa que las tasas se mantuvieron estables entre los años 2001 y 2007 para ambos sexos. Los coeficientes de la regresión PW fueron: 0,19 (IC 95\%: -0,54 a 0,$92 ; p=0,084$ ) en hombres, y -0,37 (IC 95\%: $-0,81$ a 0,$07 ; p=0,534)$ en mujeres. Al analizar la incidencia de IAM por grupo etario, se observó que la tasa aumentó en forma progresiva con la edad. En los menores de 45 años la incidencia para el período fue 6,2 por 100.000 habitantes. Para el grupo de 45 a 54 años la tasa de incidencia fue 88,$4 ; 202,4$ entre 55 y 64 años; 388,1 entre los

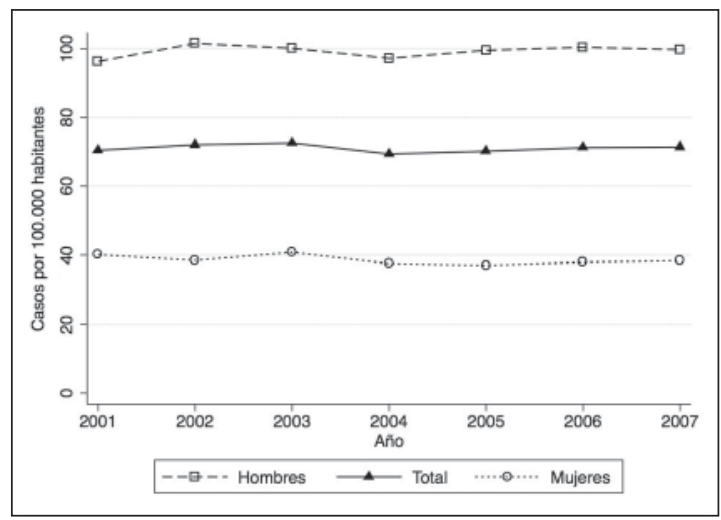

Figura 2. Tendencia de las tasas de incidencia estandarizadas de infarto agudo del miocardio según sexo en Chile, 2001 2007. Estandarizada por método directo con población estándar OMS 2000; valor p no significativo para las tendencias. 
65 y 74 años y finalmente 835,1 en los mayores de 74 años. En la Figura 3 se muestran las tasas de incidencia para los distintos grupos de edad. El análisis de la tendencia mostró un aumento de la incidencia en términos porcentuales en los grupos de menor edad: $34 \%$ en los menores de 45 años; $y$ $9,2 \%$ en el de 55 a 64 años. Por el contrario, en el grupo de 65 a 74 años se registró una reducción en la incidencia de $11,3 \%$ en el período estudiado.

El número de casos proyectados para el año
2015 es 16.041 , lo cual corresponde a un aumento de $20,6 \%$ en la frecuencia absoluta, respecto a los casos reportados en 2007 (13.277).

En cuanto a letalidad, ocurrieron en promedio 5.920 muertes por IAM anualmente, siendo la mayoría certificadas por médicos $(99,94 \%)$. La letalidad total fue $49,5 \%$, siendo significativamente mayor en mujeres $(57,2 \%$ vs $45,4 \%$; $\mathrm{p}<0,001)$. Respecto a la letalidad total, los análisis mostraron que ésta disminuyó durante el período analizado
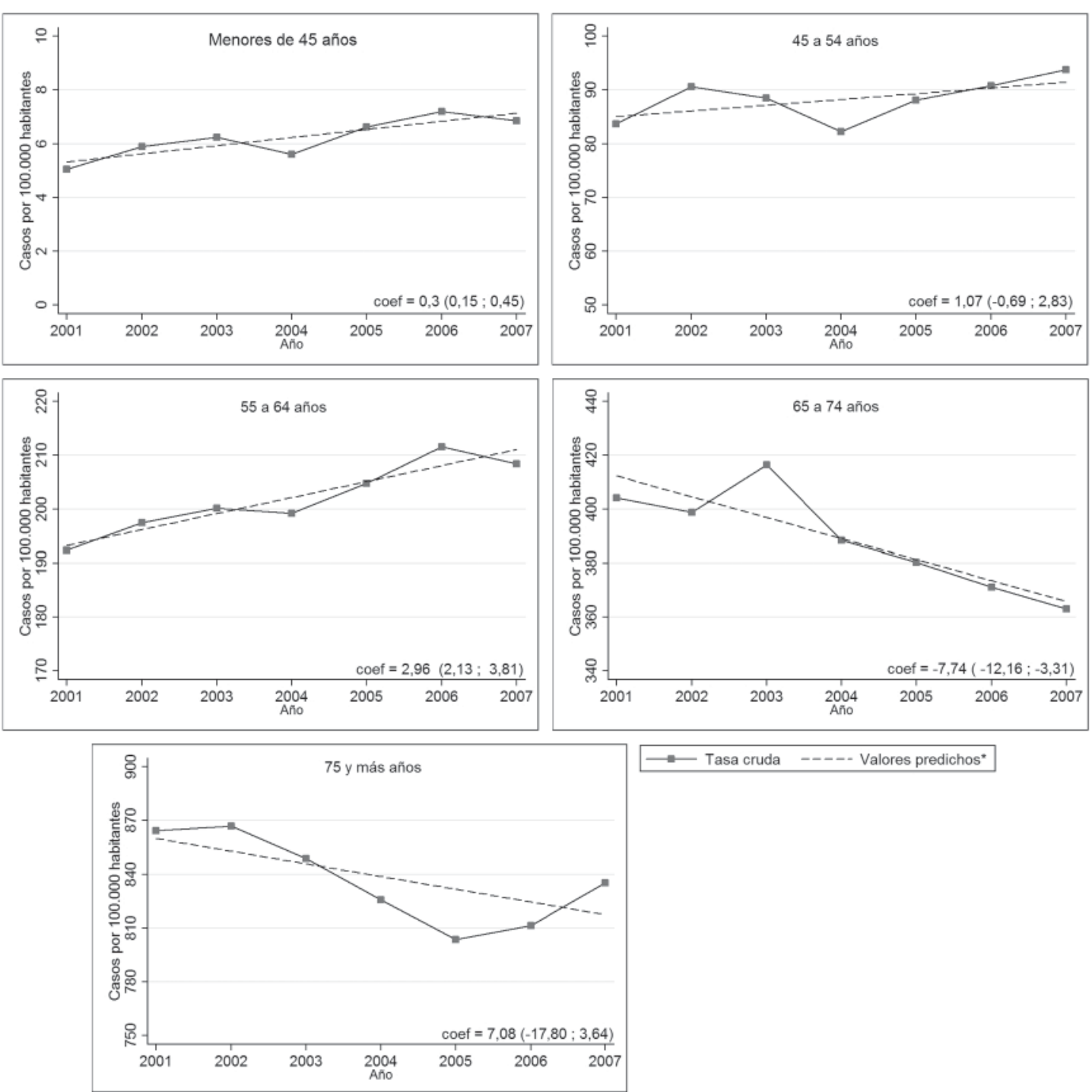

Figura 3. Tasas de incidencia de infarto agudo del miocardio según grupo de edad en Chile, 2001-2007. *Valores predichos. según modelo de Prais-Winsten. 


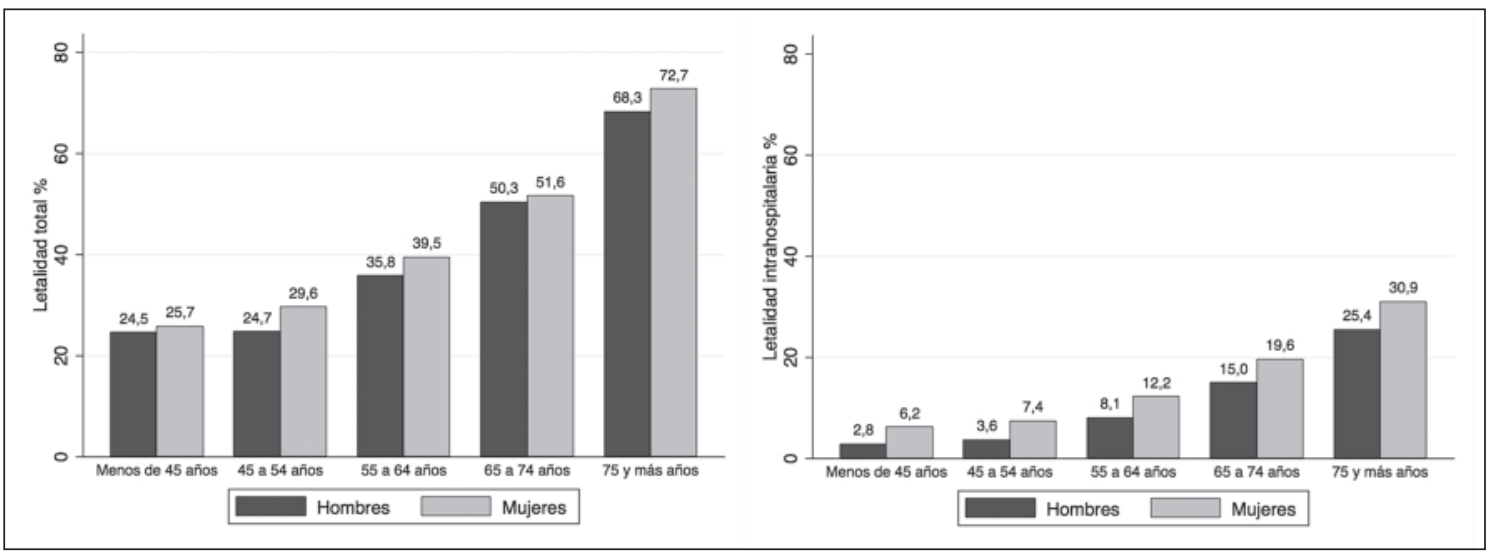

Figura 4. Tendencia de la letalidad total e intrahospitalaria de infarto agudo del miocardio según grupo de edad en Chile, 2001-2007. A) Letalidad total (\%). B) Letalidad intrahospitalaria (\%). Valor p para tendencia $<0,01$ para todos los grupos, excepto para el grupo de menores de 45 años.

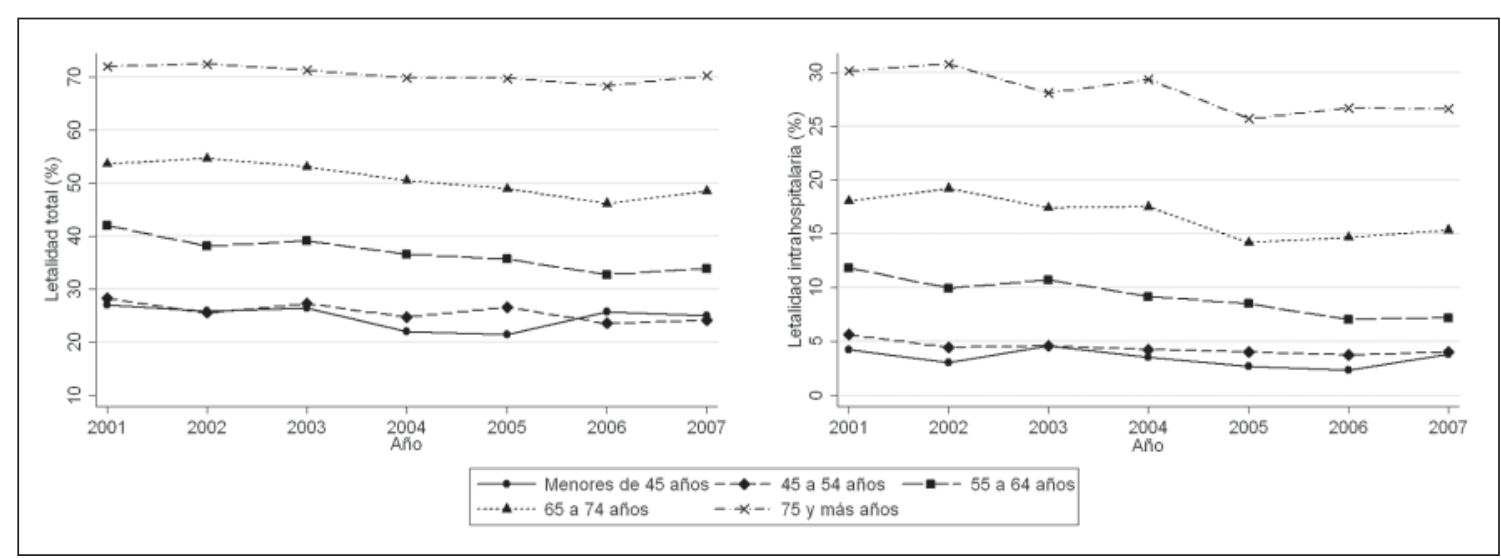

Figura 5. Letalidad total e intrahospitalaria por infarto agudo del miocardio según grupo de edad y sexo en Chile, 2001-2007 según modelo de Prais-Winsten.

en ambos sexos, particularmente en el masculino (reducción anual de 1,22\% en hombres (IC 95\%: $-1,52$ a $-0,94 ; \mathrm{p}<0,001)$ y $0,81 \%$ en mujeres (IC 95\%: $-1,25$ a $-0,37 ; \mathrm{p}=0,005)$. La letalidad intrahospitalaria fue $14,2 \%$ y también fue mayor en mujeres $(20,4 \%$ vs $11,3 \% ; \mathrm{p}<0,001)$. El análisis de tendencia mostró una reducción de $0,7 \%$ anual (IC 95\%: $-1,01$ a $-0,38 ; p=0,002$ ) y, a diferencia de la letalidad total, la disminución fue mayor en mujeres (-1,01\%; IC 95\%: -1,36 a -0,67 en mujeres y -0,57\%; IC 95\%: -0,73 a -0,41 en hombres). La Figura 4 A y B muestra la evolución de la letalidad según grupo etario. Se observó una tendencia hacia la disminución de la letalidad total e intrahospita- laria que fue significativa en todos los grupos de edad, excepto en los menores de 45 años.

En cuanto a la distribución de la letalidad total e intrahospitalaria en los pacientes con IAM según edad y sexo, ésta fue siempre mayor en mujeres y aumentó progresivamente con la edad (Figura 5).

\section{Discusión}

Los resultados muestran que Chile presentó tasas de incidencia de IAM menores a las de otros países $^{8,17}$. La incidencia fue mayor en hombres y aumentó progresivamente con la edad. Si bien las 
tasas estandarizadas por edad no mostraron un incremento en la incidencia entre 2001 y 2007, debido al progresivo envejecimiento de la población chilena $^{18}$, es esperable un aumento en el número absoluto de casos en las próximas décadas y una creciente demanda de atención para el sector salud.

Dado que las tasas de incidencia se han mantenido estables, la reducción en las tasas de mortalidad observada en Chile en la última década se explicaría principalmente por la disminución de la letalidad de los casos.

Los resultados del presente análisis muestran un aumento de la incidencia en los grupos de menor edad, siendo particularmente importante en los menores de 45 años. Esto puede deberse, en parte, a la mayor prevalencia de algunos factores de riesgo como tabaquismo, sedentarismo y sobrepeso ${ }^{19,20}$ y a la utilización de sustancias ilícitas como las anfetaminas, la cocaína o sus derivados en este grupo etario ${ }^{21}$.

La letalidad total fue cercana a $50 \%$, evidenciándose una reducción significativa en el período estudiado, similar a lo reportado por el proyecto Multinational Monitoring of Trends and Determinants in Cardiovascular Disease (MONICA) ${ }^{8}$. Los cambios en la letalidad, particularmente la intrahospitalaria, se asocian con la optimización en el tratamiento del IAM. Datos de registros internacionales, así como del Registro Chileno de Infarto Agudo al Miocardio (GEMI), han mostrado un aumento en el uso de terapia farmacológica y estrategias de reperfusión en estos pacientes, como también en la indicación de procedimientos de revascularización para el período analizado ${ }^{4,5,22}$. Esto, en parte, puede explicarse por la incorporación del IAM al Régimen de Garantías Explicitas en Salud en el año 2005(GES).

Las mujeres presentaron una mayor letalidad que puede deberse a la dificultad en el diagnóstico de IAM en ellas, la edad más avanzada de los casos y la mayor prevalencia de factores de riesgo, entre otros $^{23}$. Además, dicha diferencia podría atribuirse al tratamiento menos agresivo que reciben las mujeres durante la fase hospitalaria ${ }^{24-27}$. El registro GEMI ha reportado un menor empleo de procedimientos de reperfusión y terapias de probada eficacia en el manejo del IAM en mujeres ${ }^{23}$, a pesar que estas diferencias han disminuido en los últimos $a_{n}{ }^{22}$. Esto último se refleja en la reducción en la letalidad intrahospitalaria encontrada en mujeres.

$\mathrm{Al}$ igual que la incidencia, la letalidad aumen- tó progresivamente con la edad, siendo 3 veces superior el riesgo de muerte en los mayores de 74 años, comparado con los menores de 45 años. Estos resultados son concordantes con estudios que han identificado la edad como un factor de riesgo independiente de mortalidad en pacientes con IAM $^{28,29}$.

Entre las limitaciones de este estudio, se encuentra que la causa de muerte ha sido asignada según la información del certificado de defunción. No hay estudios a nivel nacional que validen la causa de muerte de los certificados de defunción en muertes por enfermedad coronaria, pero en estudios internacionales se ha reportado una sobreestimación en la asignación de esta causa, especialmente en sujetos de edad avanzada ${ }^{30,31}$. Esto podría producir una sobrestimación en las tasas reportadas. Sin embargo, dado que casi la totalidad de las muertes por cardiopatía coronaria ocurridas entre 1997 y 2007 fueron certificadas por médicos, se podría controlar parcialmente la introducción de este sesgo ${ }^{2}$, aunque no es posible descartarlo. Al contrario, la estimación de la incidencia podría haber sido subestimada por la falta de diagnóstico de los pacientes con IAM sin supradesnivel del segmento ST(SDST) y en pacientes diabéticos, que pueden presentar infartos silentes. Está documentado que la proporción de IAM sin SDST en el registro GEMI fue $42,6 \%$ en el sector privado y $26,3 \%$ en el sector público, lo que se asoció a un menor empleo de troponinas en este último ${ }^{32} y$, por lo tanto, a una menor probabilidad de diagnóstico.

Por otro lado, debemos mencionar que una fortaleza del presente estudio es la representatividad, dada la inclusión de la totalidad de los casos registrados en Chile. Además, se propone un método simple y factible para la estimación de tasas de incidencia de patologías que presentan una alta letalidad y/o necesidad de hospitalización, por lo que son incluidas en las bases de datos utilizadas.

En conclusión, las tasas de incidencia se han mantenido estables en la población general, sin embargo, al analizar por grupos de edad, es alarmante el aumento en personas jóvenes. Es necesario considerar el incremento de los casos en términos absolutos, ya que se requerirán mayores recursos del sector salud para responder a la demanda de atención. La letalidad ha disminuido, probablemente por una mejoría en el tratamiento de los pacientes con IAM. 


\section{Referencias}

1. Departamento de Estadísticas e Información de Salud. Estadisticas vitales Disponible en: http://deis.minsal.cl/ deis/indexmc.asp [Consultado el 2 noviembre 2010].

2. Alonso F, Nazzal C. Mortalidad por cardiopatía isquémica en Chile: quiénes, cuántos y dónde. Rev Panam Salud Pública 2010; 28 (5): 319-25.

3. Beaglehole R. International trends in coronary heart disease mortality and incidence rates. J Cardiovasc Risk 1999; 6 (2): 63-8.

4. Fox KA, Steg PG, Eagle KA, Goodman SG, Anderson FA, Jr., Granger CB, et al. Decline in rates of death and heart failure in acute coronary syndromes, 1999-2006. JAMA 2007; 297 (17): 1892-900.

5. Ford ES, Ajani UA, Croft JB, Critchley JA, Labarthe DR, Kottke TE, et al. Explaining the decrease in U.S. deaths from coronary disease, 1980-2000. N Engl J Med 2007; 356 (23): 2388-98.

6. Ministerio de Salud Gobierno de Chile. Objetivos Sanitarios para la Década 2000-2010. Evaluación final del período, grado de cumplimieto de los objetivos de impacto. In: Subsecretaría de Salud Pública/División de Planificación Sanitaria/Departamento de Epidemiología, ed. Santiago de Chile: Ministerio de Salud Gobierno de Chile.

7. Murray CJ, López AD. Alternative projections of mortality and disability by cause 1990-2020: Global Burden of Disease Study. Lancet 1997; 349 (9064): 1498-504.

8. Tunstall-Pedoe H, Kuulasmaa K, Mahonen M, Tolonen $\mathrm{H}$, Ruokokoski E, Amouyel P. Contribution of trends in survival and coronary-event rates to changes in coronary heart disease mortality: 10-year results from $37 \mathrm{WHO}$ MONICA project populations. Monitoring trends and determinants in cardiovascular disease. Lancet 1999; 353 (9164): 1547-57.

9. Corbalan R, Nazzal C, Prieto JC, Chavez E, Lanas F, Lamich R, et al. Reducción de la mortalidad por infarto del miocardio en hospitales chilenos. Rev Med Chile 2002; 130 (4): 368-78.

10. Tunstall-Pedoe H, Vanuzzo D, Hobbs M, Mahonen M, Cepaitis Z, Kuulasmaa K, et al. Estimation of contribution of changes in coronary care to improving survival, event rates, and coronary heart disease mortality across the WHO MONICA Project populations. Lancet 2000; 355(9205): 688-700.

11. Organización Mundial de la Salud. Clasificación Internacional de Enfermedades Décima Revisión. 43 Asamblea Internacional de Salud; 1990.

12. Instituto Nacional de Estadística de Chile. Proyecciones de Población de Chile 1990-2025. Gobierno de Chile, 2002.
13. Organización Mundial de la Salud. La Estandarización: Un Método Epidemiológico Clásico para la Comparación de Tasas. Boletín Epidemiológico / OPS. 2002;23(3).

14. Ahmad OB, Boschi-Pinto C, López AD, Murray CJ, Lozano R, Inoue M. Age standardization of rates: a new WHO standard. Ginebra: OMS, 2001.

15. Durbin J, Watson G. Testing for serial correlation in least squares regression. Biometrika 1950; 37(3-4): 40928.

16. StataCorp. Stata Statistical Software: Release 10. College Station. TX: StataCorp LP, 2007.

17. Yeh RW, Go AS. Rethinking the epidemiology of acute myocardial infarction: challenges and opportunities. Arch Intern Med 2010; 170 (9): 759-64.

18. Szot J. La transición demográfico-epidemiológica en Chile, 1960-2001. Rev Esp Salud Pública 2003; 77: 605-13.

19. Ministerio de Salud Gobierno de Chile. Encuesta Nacional de Salud. ENS Chile 2009 - 2010. In: Departamento de Epidemiología, ed. Santiago de Chile: Ministerio de Salud de Chile, 2010.

20. Schargrodsky H, Hernández-Hernández R, Champagne BM, Silva H, Vinueza R, Silva Aycaguer LC, et al. CARMELA: assessment of cardiovascular risk in seven Latin American cities. Am J Med 2008; 121 (1): 58-65.

21. McCord J, Jneid H, Hollander JE, de Lemos JA, Cercek $\mathrm{B}$, Hsue P, et al. Management of cocaine-associated chest pain and myocardial infarction: a scientific statement from the American Heart Association Acute Cardiac Care Committee of the Council on Clinical Cardiology. Circulation 2008; 117 (14): 1897-907.

22. Nazzal C, Campos P, Corbalán R, Lanas F, Bartolucci J, Sanhueza P, et al. Impacto del plan AUGE en el tratamiento de pacientes con infarto agudo al miocardio con supradesnivel ST, en hospitales chilenos. Rev Med Chile. 2008; 136 (10): 1231-9.

23. Prieto JC, Chavez E, Corbalán R, Yovanovich J, Cumsille F, Nazzal C. Infarto agudo del miocardio en Chile: diferencias entre hombres y mujeres en su evolución y pronóstico. Rev Med Chile 1996; 124 (7): 785-92.

24. Nante N, Messina G, Cecchini M, Bertetto O, Moirano F, McKee M. Sex differences in use of interventional cardiology persist after risk adjustment. J Epidemiol Community Health 2009; 63 (3): 203-8.

25. Jneid H, Fonarow GC, Cannon CP, Hernández AF, Palacios IF, Maree AO, et al. Sex differences in medical care and early death after acute myocardial infarction. Circulation 2008; 118 (25): 2803-10.

26. Heer T, Gitt AK, Juenger C, Schiele R, Wienbergen H, Towae F, et al. Gender differences in acute non-STsegment elevation myocardial infarction. Am J Cardiol 2006; 98 (2): 160-6. 
27. Anand SS, Xie CC, Mehta S, Franzosi MG, Joyner C, Chrolavicius S, et al. Differences in the management and prognosis of women and men who suffer from acute coronary syndromes. J Am Coll Cardiol 2005; 46 (10): 1845-51.

28. Gale CP, Manda SO, Batin PD, Weston CF, Birkhead JS, Hall AS. Predictors of in-hospital mortality for patients admitted with ST-elevation myocardial infarction: a real-world study using the Myocardial Infarction National Audit Project (MINAP) database. Heart 2008; 94 (11): 1407-12.

29. Lanas F, Nazzal C, Corbalán R, en representación del grupo GEMI. Sobrevida precoz y alejada en pacientes post infarto: efecto de la edad. Rev Chil Cardiol 2009; 29: 344.
30. Lloyd-Jones DM, Martin DO, Larson MG, Levy D. Accuracy of death certificates for coding coronary heart disease as the cause of death. Ann Intern Med 1998 15; 129 (12): 1020-6.

31. Coady SA, Sorlie PD, Cooper LS, Folsom AR, Rosamond WD, Conwill DE. Validation of death certificate diagnosis for coronary heart disease: the Atherosclerosis Risk in Communities (ARIC) Study. J Clin Epidemiol 2001; 54 (1): 40-50.

32. Corbalán R, Nazzal C, Sanhueza P, Campos P, Lanas F, Cavada G, et al. Utilización de troponinas en hospitales chilenos y su importancia en el diagnóstico de infarto del miocardio sin elevación del segmento ST. Rev Chil Cardiol 2009; 28: 361. 